\title{
European Stroke Science Workshop
}

\author{
Heinrich P. Mattle ${ }^{a}$ Michael Brainin ${ }^{b}$ Angel Chamorro ${ }^{c}$ Hans Christoph Diener ${ }^{d}$ \\ Werner Hacke ${ }^{e}$ Didier Leys ${ }^{f}$ Bo Norrving ${ }^{g}$ Nick Ward ${ }^{h}$ \\ ${ }^{a}$ Department of Neurology, Inselspital, University of Bern, Bern, Switzerland; bepartment for Clinical \\ Neurosciences and Preventive Medicine, Danube University Krems, Krems, Austria; ${ }^{C}$ Institute of Neurosciences, \\ Hospital Clinic de Barcelona, University of Barcelona, Barcelona, Spain; ${ }^{d}$ Department of Neurology, University \\ Hospital Essen, Essen, and 'Department of Neurology, University of Heidelberg, Heidelberg, Germany; \\ ${ }^{f}$ Department of Neurology and Stroke Unit, Lille University Hospital, Hôpital Roger Salengro, Lille, France;

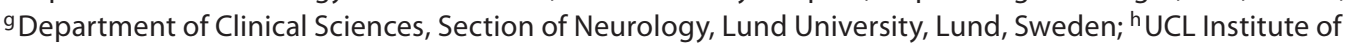 \\ Neurology, Queen Square, London, UK
}

\section{Key Words}

Biomarkers · Cerebral reorganization $\cdot$ Immunology • Hypothermia $\cdot$ Reperfusion $\cdot$ Stem cell therapy $\cdot$ Stroke

\begin{abstract}
The European Stroke Organisation held its first European Stroke Science Workshop in Garmisch-Partenkirchen, Germany (December 15-17, 2011). Stroke experts based in Europe were invited to present and discuss their current research. The scope of the workshop was to review the most recent findings of selected topics in stroke, to exchange ideas, to stimulate new research, and to enhance collaboration between European stroke research groups. Seven scientific sessions were held, each starting with a keynote lecture to review the state of the art of the given topic, followed by 4 or 5 short presentations by experts. They were asked to limit their presentations to 10 slides containing only recent information. The meeting was organized by the executive committee of the European Stroke Organisation (Heinrich Mattle, chairman, Michael Brainin, Angel Chamorro, Werner Hacke, Didier Leys) and supported by the European Stroke Conference (Michael Hennerici). The following sections summarize the content of the workshop.
\end{abstract}

Copyright $\odot 2012$ S. Karger AG, Basel (c) 2012 American Heart Association, Inc., and S. Karger AG, Basel 1015-9770/12/0342-0095\$38.00/0

Accessible online at:

www.karger.com/ced

\section{Session I: The Brain and Immune System Interplay in Acute Stroke}

\section{Ángel Chamorro Reviewed Recent Studies on the Benefits and Harms of the Innate and Adaptive Immune Response after Acute Stroke}

Ángel Chamorro reviewed the recent clinical and experimental studies on the benefits and harms of the innate and adaptive immune response after acute stroke [1]. The Toll-like receptors and the lectin pathway of complement activation were identified as innate amplifiers of the inflammatory cascade after stroke. The dynamics of different populations of lymphocytes in stroke were described, including the effects of T cells and B cells on different neural cells, the controversial effects of regulatory $\mathrm{T}$ cells, and the role of regulatory B cells. Recently reported mechanisms of infection after acute stroke include a noradrenaline-mediated activation of hepatic invariant natural killer $\mathrm{T}$ cells and release of acetycholine in the spleen by $\mathrm{T}$ memory cells. Additional recent studies in

This article has been co-published in Stroke and Cerebrovascular Diseases. 
stroke patients show an increased presence of neuronal and myelin antigens in lymph nodes of the lymphatic drainage of the central nervous system [2]. The cellular source of the neural antigens disclose antigen-specific autoimmune responses, either protective (neurons) or deleterious (myelin). Collectively, these data emphasize the double-edged nature of the crosstalk between the brain and the immune system, and the strong need for further translational research to unravel the role of different $\mathrm{T}$ cell populations to damaging and reparative processes that follow acute stroke.

\section{Roland Veltkamp, Heidelberg, Germany, Discussed Roles of Immunodepression vs. Immunomodulation after Ischemic Stroke}

Roland Veltkamp (Heidelberg, Germany) discussed the roles of immunodepression vs immunomodulation after ischemic stroke, including a decrease of the number and activity of immune cells, which results in an increased risk of infections. Hallmarks of this response are decreased function of monocytes and lymphocytopenia. Suppression of the antibacterial surveillance function of immune cells after stroke in humans and rodents results primarily from extensive brain infarction, whereas the immune system alterations are more subtle after smaller infarctions. There is a shift toward more regulatory $\mathrm{T}$ cells, which modulates the activity of microglia and the activation of invading $\mathrm{CD}^{+} \mathrm{T}$ cells. Inhibition of the leukocyte adhesion molecule $\alpha 4$-integrin or its endothelial counterpart vascular cell adhesion molecule to reduce brain injury in moderate ischemia was also discussed [3] Current evidence also suggests a major role for the sympathetic system, the hypothalamic-pituitary-adrenal axis, and the vagal nerve in facilitating the crosstalk between the injured brain and the immune system, although the signals underlying their activation and dysfunction remain to be better-elucidated.

\section{Andreas Meisel, Berlin, Germany, Addressed the Immune System in Subarachnoid Hemorrhage}

Comparable to acute central nervous system injury and ischemic stroke [4], patients with subarachnoid hemorrhage have development of a temporary but rapid immunodepression [5]. Twenty percent of these patients have development of pneumonia. Within $24 \mathrm{~h}$ of subarachnoid hemorrhage, patients have development of lymphopenia of $\mathrm{CD} 4^{+} \mathrm{T}$ cells, $\mathrm{CD} 8^{+} \mathrm{T}$ cells, and NK cells, and a diminished release of proinflammatory cytokines involved in antibacterial defense, such as lymphocytic interferon- $\gamma$ and monocytic tumor necrosis factor- $\alpha$. Magnitude and duration of the immunodepression correlate with the severity of subarachnoid hemorrhage. Whereas in asymptomatic patients immunity is recovered within 3 to 5 days, symptomatic patients with delayed cerebral ischemia have development in persistent immune suppression. Persistent immunodepression is strongly correlated with subarachnoid hemorrhage-associated pneumonia. Markers such as monocyte human leukocyte antigen-DR expression might help target those at greatest risk.

\section{Xabier Urra, Barcelona, Spain, Summarized the Role of Monocytes in Acute Stroke}

The number of monocytes reaching the human brain increases after stroke but little is known about their function. Flow cytometry in stroke patients shows a reduced expression of antigen-presenting molecules and greater expression of Toll-like receptor- 4 . After ex vivo stimulation with lipopolysaccharide, monocytes show a reduced capacity to produce tumor necrosis factor- $\alpha$, whereas the production of interleukin-10 is maintained [6]. Poor prognosis after stroke is associated with higher expression in monocytes of Toll-like receptor- 4 and predominance of classic inflammatory monocytes over minor populations of reparative $\mathrm{CD} 16^{+}$monocytes. Infections are associated with a low expression of antigen-presenting molecules, costimulatory signals, and adhesion molecules, and a lower production of tumor necrosis factor- $\alpha$.

\section{The Prevention of Infection in Acute Stroke Was Addressed by Diederik van de Beek, Amsterdam, The Netherlands}

Several studies have evaluated the preventive use of antibiotics in patients with acute stroke with conflicting results. In a systematic overview and meta-analysis of randomized clinical trials, preventive antibiotics reduced the risk of infection but did not reduce mortality [7]. In the NeuMast (NCT00930020), 330 patients with ischemic stroke will be randomized between minocycline or placebo; 165 patients have been included and an interim analysis is being performed. The second trial (ISRCTN37118456) is a pragmatic cluster randomized trial with a blinded endpoint (chest infections). Patients 
( $\mathrm{n}=800)$ with stroke plus swallowing disorder have been included and preventive use of amoxicillin and clarithromycin is compared with standard care. Finally, the Preventive Antibiotics in Stroke Study (ISRCTN66140176), a multicenter, prospective, randomized, open-label, blinded endpoint trial, investigates whether the preventive use of ceftriaxone improves functional outcome in patients with stroke. As of now, $\approx 800$ of the aimed 3,200 patients have been included.

\section{Session II: Hypothermia and Neuroprotection}

\section{Ulrich Dirnagl from Berlin Addressed the Question, Why Did All Clinical Neuroprotectant Trials Fail?}

The translation from bench results is frequently irrational and limited preclinical data are used to rush into clinical development [8]. Inadequate models often are used and trial design suffers from bias, low power, small sample sizes, lack of randomization and blinding, inadequate time windows, and incorrect dosages. A major issue remains the difference between uniform strokes in experimental juvenile animals vs heterogeneity in human stroke. He also highlighted the complexity of pathophysiology of brain ischemia and expanded on the fact that targeting a highly elective single mechanism like 1 transcription factor or a single channel will not succeed in clinical trials. Agents should be pleiotropic. His conclusion was that neuroprotection is not dead and translation may work if preclinical research quality is improved and clinical development is robust and takes care of time effects, selection of patients, and surrogate endpoints in early development.

\section{Bart van der Worp, Utrecht, Holland, Opened the Discussion of Hypothermia in Acute Ischemic Stroke}

Bart van der Worp from Utrecht, Holland, opened the discussion of hypothermia in acute ischemic stroke by introducing the basic design of a large randomized clinical trial, EuroHYP-1, to be funded by the European Union as part of the FP7 Program. It will include awake patients with acute ischemic stroke who will be cooled to a target temperature of $34-35^{\circ} \mathrm{C}$ within $6 \mathrm{~h}$ after symptom onset. Cooling will be maintained for $24 \mathrm{~h}$ and outcome will be tested at 3 years using the modified Rankin scale. Cooling will be initiated with an intravenous infusion of 20 $\mathrm{ml} / \mathrm{kg}$ cooled normal saline over 30-60 min (temperature $4^{\circ} \mathrm{C}$ ), followed by either surface cooling or endovascular cooling for $24 \mathrm{~h}$. Because it is impossible to perform this in a double-blind session, assessors will be blinded to treatment allocation at 3 months (probe design). The trial will have 750 patients per arm and is designed to show a 7\% absolute improvement and a 5\% significance level.

\section{Derk Krieger, Copenhagen, Denmark, Summarized the Experience of Therapeutic Hypothermia in Spontaneous Intracerebral Hemorrhage}

Derk Krieger out that few experimental models had approached this problem. Although there has been some effect on hemorrhage-associated edema, clinical benefit has been rarely reported. A recent clinical pilot study from Erlangen on therapeutic hypothermia in patients with large symptomatic intracerebral hemorrhage compared with historic matched controls did not show any major differences in outcome. In summary, the experimental and clinical evidence for hypothermia in acute ischemic stroke is scarce and does not yet suggest the design of a large randomized clinical trial.

\section{Jesper Petersson, Lund, Sweden, Reviewed New Devices and New Routes for Hypothermia}

He described preclinical and clinical use of hypothermia. He also reviewed the different devices used for body surface cooling like pads and blankets and intravasal whole-body cooling with vena cava catheters of cold fluid infusion. A major subset of new devices is designed for local cooling, intranasal cooling, head caps, or ice-cold pads. The side effects of shivering and pain are still problematic. At $<34^{\circ}$, patients usually have to be fully anesthetized. Methods to prevent shivering in awake patients include sedation with Meperidine (eg, $0.25 \mathrm{mg} / \mathrm{kg} / 1$ hour intravenous) with oral Buspirone $20 \mathrm{mg}$ every $8 \mathrm{~h}$.

\section{Katja Piironen, Helsinki, Finland, Gave an Update on Hypothermia after Cardiac Arrest and Brain Trauma}

Meta-analyses and systematic reviews give no firm support for a beneficial effect of hypothermia on mortality and outcome after trauma. A number of issues in this field are still unresolved, including depth and duration of hypothermia. 
The situation is better for hypothermia after cardiac arrest. According to a Cochrane database meta-analysis in 2009, 'hypothermia seems to improve hospital discharge and neurological outcome after cardiac arrest.' Other systematic reviews come to similar conclusions, although there is still a lack of high-level clinical randomized trials. Some randomized controlled trials comparing moderate hypothermia $\left(33^{\circ} \mathrm{C}\right)$ to very mild hypothermia $\left(36^{\circ} \mathrm{C}\right)$ are currently ongoing.

Despite the fact that hypothermia after cardiac arrest has received high-level recommendations in recent guidelines, there is still plenty of room for improvement. Again, as in drug development in which dose-finding is a key issue, deaths from hypothermia, duration of hypothermia, and strategy of rewarming in hypothermia are still issues that need to be solved.

\section{Session III: Stem Cell Therapy for Stroke}

\section{Zaal Kokaia, Lund, Sweden, Discussed Cellular Plasticity after Stroke: Important for Recovery?}

Stem cell-based approaches hold much promise as potential novel treatments to restore function after stroke. Transplantation of stem cells or their derivatives in animal models can improve function by replacing the neurons and glia cells lost (as shown in neurodegenerative diseases) and through trophic actions, and by modulation of inflammation. Endogenous neural stem cells also are potential therapeutic targets because they produce neurons and glial cells in response to injury. Clinical trials are ongoing but there are significant hurdles before basic research findings can be responsibly translated to novel therapies. In particular, there is a need to betterunderstand mechanisms of action of stem cells after transplantation in stroke-lesioned brain and learn how to control proliferation, survival, migration, and differentiation in the pathological environment of grafted and endogenously derived stem cells.

\section{Gianvito Martino, Milan, Italy, Reported on Interactions between Stem Cells and the Immune System}

Apart from a cell replacement mechanism, neural stem/ precursor cell (NPC) transplantation also may promote central nervous system repair via intrinsic neuroprotec- tive bystander capacities. At the site of tissue damage, undifferentiated stem cells release neuroprotective molecules (eg, immunomodulatory substances, neurotrophic growth factors, and stem cell regulators), which are also normally expressed by NPC for maintaining tissue homeostasis. The ability of transplanted NPC to protect the brain from several types of injuries using bystander strategies ('therapeutic plasticity') is of pivotal importance for the future of stem cell-based therapeutic approaches.

\section{Mathias Hoehn, Cologne, Germany, Presented Neuroimaging Aspects on Stem Cell Mediation of Functional Recovery after Stroke}

Cell migration from the implantation site may be followed with in vivo magnetic resonance imaging (MRI) using responsive contrast agents, and cell-specific differentiation processes may be visualized. Functional MRI activation studies have demonstrated an unequivocal therapeutic improvement after several months indicating that regeneration studies in rodents require longitudinal investigation protocols. The imaging-based regeneration investigations need to be extended beyond the focused task-selective brain activation to analyze brain connectivity using resting-state functional MRI. Combining resting-state functional MRI with fiber tracking, disturbances of structural and functional networks are now investigated in longitudinal approaches.

\section{Robert Andres, Bern, Switzerland, Presented Further Aspects on Intravascular Stem Cell Therapy for Stroke}

Because ischemic stroke commonly affects wide brain areas, intravascular delivery of NPC may be a more promising approach than focal intraparenchymal transplantation. NPC administered into the carotid artery selectively pass the blood-brain barrier at the infarct site, exhibit neural differentiation, and improve functional deficits in rodent models. Compared with intravenous injection, intra-arterial administration is a more efficient route, allowing first-pass delivery of the cells to the brain. Critical aspects to be addressed include the limited transendothelial homing of NPC, as well as biodistribution and safety issues. 


\section{Keith Muir (Glasgow, United Kingdom) Reported that Designs of Human Trials of Stem Cell Therapy Will Be Dictated by Preclinical Evidence (Delivery Routes/Timings, Cell Properties) and Also by Regulatory Perspectives}

Safety trials of cell therapies have used direct intraparenchymal brain injection of allografts or xenografts (3 completed trials, 2 ongoing) in chronic cases between 6 months and several years after stroke or intravenous delivery of autologous stem cells in the subacute phase. The design of Pilot Investigation of Stem Cells in Stroke (PISCES) trial (ongoing, recruitment target expected during 2012), which uses genetically modified human neural stem cells delivered by intraparenchymal injection, was presented.

\section{Bo Norrving, Lund, Sweden, Presented a Cost-Effectiveness Study on Stem Cell Therapy after Stroke}

The study assumptions included direct and indirect costs and prognostic data, and modeling was performed for age, functional status at discharge, degree of therapeutic effectiveness, and 4 other variables. The study showed that stem cell therapy offers potential for cost offset and cost-savings in the long-term by reducing disability after stroke. The treatment appeared cost-effective under a wide range of assumptions.

\section{Session IV: Biomarkers and Stroke}

\section{Joan Montaner, Barcelona, Spain, Discussed Biomarkers in Stroke}

The future value of biomarkers in clinical practice might be: (1) to predict stroke risk; (2) to make stroke diagnosis; (3) to differentiate stroke subtypes; (4) to establish outcome; and (5) to use biomarkers as treatment endpoints. In addition, biomarkers may help to understand the pathophysiology of stroke, and specific brain biomarkers might aid in stroke diagnosis and permit rapid referral of stroke patients to stroke centers for acute treatments including thrombolysis [9]. To date, diagnostic biomarkers such as b-nerve growth factor, interleukin-17, tumor necrosis factor receptor-1, and insulin-like growth factor-binding protein were shown to differentiate acute strokes from stroke mimics. Biomarkers also might yield information on evolution and prognosis of stroke that is not contained in the clinical data. Such biomarkers include markers of infarct growth, hemorrhagic transformation, cardiac complications, infections, or recurrent vascular events. They might help to weigh the potential risks and benefits of different treatments and to allocate resources for stroke treatment. However, whether these putative biomarkers will have direct clinical utility is currently unclear.

\section{Patrik Michel, Lausanne, Switzerland, Discussed the Role of Imaging As a Marker of Viable Tissue}

Although 'time is brain' holds true for all acute recanalization treatments in ischemic stroke, patient and method selection is now shifting toward hyperacute assessment and effective treatment strategies for the occluded vessel ('recanalization is brain') and of viable tissue ('penumbra is brain'). In clinical practice, the latter is best assessed by magnetic resonance perfusion-weighted imaging or computed tomography perfusion. Thresholds to differentiate nonviable from viable tissue and from benign oligemia now have been better-defined, and automated threshold maps are available [10]. Randomized trials based on such viable tissue determination ('mismatch') have started (DEFUSE-2, EXTEND) or are planned (ECASS-4).

\section{Hugues Chabriat, Paris, France, Spoke on Biomarkers of Small Vessel Disease}

Hugues Chabriat emphasized some of the most recent developments on MRI markers of small vessel disease in the literature: (1) first report on predictors of incident microbleeds in the general population; (2) blood products within pericytes and erythrophagocytosis as potential mechanisms underlying these lesions; (3) accumulating evidence of blood-brain barrier alterations in small vessel disease using dedicated MRI sequences; (4) increased knowledge of the wide spectrum of clinical manifestations related to diffuse white-matter changes; (5) further confirmation that blood pressure reduction can reduce the progression of white matter hyperintensities in a populationbased study; (6) dilated perivacular spaces reported as an independent MRI marker of small vessel disease related to male gender, age, and hypertension with their severity associated with increased risk of incident dementia; and (7) 
limitations of MRI diagnostic criteria of so-called silent infarcts and recent detection of cortical microinfarct in small vessel disease using ultrahigh-resolution MRI.

\section{Hugh Markus, London, United Kingdom, Discussed Genetic Markers of Stroke Risk}

Identifying genetic risk factors for stroke may help in both risk prediction in individual patients and in identifying new stroke mechanisms that allow new therapeutic approaches to be developed. The genome-wide association study approach allows $>1$ million markers spanning the genome to be compared between cases and controls. This technique does not require any priori hypothesis and, therefore, completely novel associations can be detected. Genome-wide association study has had a major impact on genetics of other complex diseases, with $>1,000$ new associations reported by the second quarter of 2010. Initial studies in stroke confirmed that genes associated with both atrial fibrillation and myocardial infarction also were risk factors for stroke. More recently, the Wellcome Trust Case Control Consortium 2 (WTCCC2) study has looked at $>10,000$ stroke cases and 50,000 controls and identified a novel association at $7 \mathrm{p} 21$, which was specific to large artery stroke [11]. The likely underlying gene is histone deacetylase 9. There are a number of potential mechanisms by which altered histone deacetylase 9 activity could increase stroke risk. All the genome-wide association studies in stroke to date have found associations with specific stroke subtypes. This emphasizes that the pathophysiology of different subtypes differs, and this also may have implications for therapeutic responses. It also emphasizes that for the maximal chance of success in genetic studies in stroke, cases should be carefully phenotyped.

\section{Christian Foerch, Frankfurt, Germany, Spoke on Early Differentiation of Hemorrhage and Ischemia by Biomarkers}

The BE FAST study aimed to determine the diagnostic accuracy of glial fibrillary acidic protein as a biomarker of intracerebral hemorrhage in a prospective and multicenter setting [12]. Patients suspected of having acute hemispheric stroke were included. Glial fibrillary acidic protein plasma levels at admission were increased significantly in patients with intracerebral hemorrhage in comparison with patients with ischemic stroke. The chosen cut-off level provided a diagnostic sensitivity of $84 \%$ and a diagnostic specificity of $96 \%$ for differentiating intracerebral hemorrhage from ischemic stroke and stroke mimics. Further studies are needed to test a glial fibrillary acidic protein point-of-care system that may allow an optimized prehospital management of patients with symptoms of acute stroke.

\section{Session V: Anticoagulation and Reperfusion}

\section{Didier Leys, Lille, France, 'Ischemic Stroke in Adults and Genes Interfering with Coagulation, Platelet Activation, and Thrombolysis'}

In the keynote lecture, 'Ischemic Stroke in Adults and Genes Interfering With Coagulation, Platelet Activation, and Thrombolysis,' Didier Leys (Lille, France) covered the diagnostic work-up of 'cryptogenic' ischemic strokes in young adults. The work-up often includes tests for thrombophilia, although a causal relationship to stroke has never been established. Besides, genetic factors influence efficacy and safety of drugs acting on coagulation, platelets, or thrombolysis, but whether they should be used in practice remains uncertain. The association between arterial ischemic stroke and genes interfering with coagulation, platelet activity, and thrombolysis was reviewed. Most studies on coagulation disorders suffered severe methodological limitations, especially small sample size and publication bias. There is no proof for a causal relationship between any coagulation disorder and the risk of ischemic stroke. However, a small effect may exist for prothrombin G20210A mutation in adults and protein $\mathrm{C}$ deficiency in children. A single case-control study suggests an association between ischemic stroke and (high) von Willebrand factor activity, without evidence for polymorphisms in genes encoding for platelet receptors or for genes interfering with thrombin formation and fibrinolysis (fibrinogen, factor XIIIa activity, thrombin-activatable fibrinolysis inhibitor). The influence of genetic factors on the efficacy and safety profile of aspirin, clopidogrel, or vitamin $\mathrm{K}$ is proven, but the practical interest requires trials to evaluate whether a personalized prescription provides a clinical benefit. Genetic thrombophilia plays a very small role, if any, in the risk of ischemic stroke. Screening is not cost-effective and may lead to inappropriate prescriptions. Large, prospective, population-based studies, as well as trials with personalized design vs. fixed doses of clopidogrel or warfarin are needed. 


\section{Atrial Fibrillation and Stroke Prevention Was Addressed by Hans-Christoph Diener, Essen, Germany}

Oral anticoagulation with vitamin $\mathrm{K}$ antagonists (warfarin, phenprocoumon) is successful in both primary and secondary stroke prevention in patients with atrial fibrillation, yielding a $60-70 \%$ relative reduction in stroke risk and $26 \%$ relative reduction in mortality compared with placebo. Acetylsalicylic acid reduces the relative risk of stroke by a nonsignificant $19 \%$ compared with placebo, and increased bleeding risk offsets any therapeutic gain from the combination of acetylsalicylic acid with clopidogrel. A number of new drugs for oral anticoagulation that do not exhibit the limitations of vitamin $\mathrm{K}$ antagonists have been investigated. These include direct factor Xa inhibitors (rivaroxaban, apixaban) and direct thrombin inhibitors (dabigatran). Recent studies (RE-LY, ROCKET-AF, AVERROES, ARISTOTLE) showed higher efficacy and significantly lower incidence of intracranial bleeds compared with warfarin. Dabigatran in high doses prevents ischemic strokes in patients with atrial fibrillation. Rivaroxaban showed efficacy over warfarin in patients at high risk with a high $\mathrm{CHADS}_{2}$ score. Apixaban showed the lowest rate of major bleeding complications. Apixaban also showed clear superiority compared with aspirin in patients with atrial fibrillation unsuitable for a treatment with warfarin with a comparable bleeding rate. The new substances showed similar results in secondary as in primary stroke prevention in patients with atrial fibrillation.

\section{Gerhard Schroth, Bern, Switzerland, Reported on Advances in Mechanical Recanalization and Presented New Devices and Techniques}

Strokes of National Institutes of Health Stroke Scale score $\geq 12$ show large vessel occlusions (carotid T, M1) in $>90 \%$, resulting in low recanalization rates after intravenous thrombolysis. Recent improvements in endovascular therapy result in recanalization rates up to $90 \%$, corresponding with improved clinical outcome [13]. One hundred forty-one patients with severe stroke (National Institutes of Health Stroke Scale score 18) were treated in 6 European centers using the Solitaire Retrievable Stent System with recanalization rates of $90 \%$ and good clinical outcome (modified Rankin Scale score, $0-2$ ) of 55\% or even $66 \%$ in a subgroup treated with intravenous recombinant tissue plasminogen activator before mechan- ical recanalization (bridging therapy). This indicates that severe strokes may be candidates for endovascular therapy. Prospective randomized trials are urgently needed not only to show high rates of recanalization with stent retrievers but also to show superiority in clinical endpoints.

\section{Werner Hacke, Heidelberg, Germany, Reported on Clinical Endpoint Studies}

At present, up to $25 \%$ of all unselected patients with acute ischemic stroke may be candidates for device-assisted recanalization therapy. In recent studies, patient composition varied largely between reported series. Carotid Tocclusions remain difficult to treat and M1 occlusions are ideal for recanalization with devices. M2 occlusions also might show good results with intravenous thrombolysis. Occlusions of the basilar artery need to be investigated in separate studies. Until now in prospective and retrospective case series, best outcomes were reported with early recanalization. Several series reported late onset of treatment, up to $\geq 8 \mathrm{~h}$. Individual reports indicate not only delayed start of recanalization but also procedures $>4$ or $5 \mathrm{~h}$ with several devices used in sequence. Time to recanalization may be $>10 \mathrm{~h}$.

Recanalization rates with devices range between $42 \%$ and $90 \%$. To date there is no evidence that clinical outcome is improved by mechanical recanalization. Registries and trials with historical controls report low modified Rankin Scale scores of 0-2 for response rates, high mortality rates, and relatively high spontaneous ICH rates. This may be an adequate result for the study population tested, but without concurrent control this remains unknown.

After encouraging data from IMS I and IMS II, IMS III is the pivotal trial with $>600$ of 900 planned patients included. The IMS III has a 2:1 randomization between intervention and systemic thrombolysis, uses many types of transvascular therapy including intra-arterial lytics vs intravenous recombinant tissue plasminogen activator (40 minutes, $0.9 \mathrm{mg} / \mathrm{kg}$ ), and uses treatment within $6 \mathrm{~h}$ with the following devices: PENUMBRA, MERCI, SOLITAIRE, and PHENOX.

In the future there will be a number of prospective randomized trials comparing recanalization devices with intravenous or intra-arterial thrombolytic therapy. These trials will show us whether recanalization with devices improves clinical outcome in patients with severe strokes. 
José M. Ferro, Lisbon, Portugal, Discussed Advances in Treatment of Cerebral Venous Thrombosis

Four topics were addressed: type of anticoagulation, duration of anticoagulation local thrombolysis, and decompressive surgery. In the acute phase, low-molecularweight heparin seems to be safer and preferable to intravenous heparin. The duration of anticoagulation to prevent recurrent venous thrombotic events after cerebral venous thrombosis is uncertain. It will be investigated in a randomized clustered trial (EXCOA-CVT), which will compare 3 vs. 12 months of anticoagulation. Local intrasinus thrombolysis is being investigated in the TO-ACT trial, which is randomizing severe cerebral venous thrombosis patients between local thrombolysis and anticoagulation. The life-saving role of decompressive surgery in cerebral venous thrombosis patients with large herniating lesions will be investigated in the DECOMPRESS registry.

\section{Session Vl: Population-Based Interventions}

\section{Jaakko Tuomilehto, Helsinki, Finland, Gave a Keynote Lecture on Population-Based Interventions Tested for Community Implementation}

Comparing community approaches to individual approaches of prevention, Tuomilehto pointed out that in community approaches the preventive dose is viable and outcomes differ from medical outcomes. Among the prerequisites are that it has to be demonstrated that the intervention is working. This can be shown most efficiently using randomized controlled trials. However, randomized controlled trials may not give us the right answer for real life or for all people because randomized controlled trials always rely on specially selected and small number of people. There are 2 potential types of effect modification, referred to as 'behavioral effect modification,' and the impact indicator, referred to as 'biological effect modification.' Prevention looks at healthy lifestyle vs risk factors with the aim to reduce stroke events. One Finnish study has shown that a reduction of healthy lifestyle traits to $<3$ healthy lifestyle factors increases the hazard ratio for stroke from $1.37(1.02-1.83)$ to 2.08 (1.56-2.70) in hypertensive patients treated with antihypertensive drugs [14]. This illustrates the need to also give attention to lifestyle issues even in a situation when evidence-based pharmacotherapy is given to the hypertensive patients to prevent stroke.

Nonsmoking regulations, reduction of butter consumption, increase in the use of vegetable oil, and reduc- tion of salt intake with a target of $5 \mathrm{~g} /$ day have massive effects on vascular mortality including stroke. Globally, the reduction of salt intake by $5 \mathrm{~g} /$ day from the present level alone would result in a decrease in cardiovascular disease mortality by $17 \%$ and a decrease in stroke mortality by $23 \%$. On a global scale this would imply, every year, $1,265,000$ less people with strokes and 3,000,000 less people with cardiovascular deaths, overall a reduction of almost 5 million deaths per year [15].

Cerebrovascular disease rates, both incidence and case fatality, are particularly strongly influenced by socioeconomic factors. Low-income populations show a 2-times higher incidence of stroke than high-income populations within the same country. Reduction of the burden of stroke was recently stressed in the American Guideline [16], and a European Guideline also should aim at showing the returns for prevention of stroke.

\section{Laszlo Csiba, Debrecen, Hungary, Reported the Needs and Numbers for Population Interventions}

He reported a focus on Eastern Europe as opposed to the countries in Western Europe and showed that the extent and numbers of risk factors were much higher in eastern Europe according to the World Health Organization Europe July 2011 data. Comparing these countries, there was a 3-fold difference for admission rates of stroke, but life expectancy was not so strongly influenced by socioeconomic levels. Similar rates were seen for DALYS, showing that the strongest burden of stroke was in males between ages 45 and 60 years and in females older than 70 years.

\section{Philip Bath, Nottingham, United Kingdom, Talked about a Randomized Controlled Trial That Aims to Reduce Poststroke Cognitive Decline and Dementia by Strict versus Guideline Blood Pressure and/or Lipid Lowering}

Philip Bath, Nottingham, United Kingdom, in his lecture on drug compliance and adherence trials, talked about a randomized controlled trial (PODCAST, ISRCTN85562386; http://www.podcast-trial.org/) that aims to reduce poststroke cognitive decline and dementia by strict vs. guideline blood pressure and/or lipid lowering. He discussed the numbers needed to meet these endpoints and showed some preliminary (blinded) group comparisons from his trial. 


\section{Karl Matz, Krems, Austria, Addressed}

Polyintervention in the Ongoing ASPIS Trial

Karl Matz, Krems, Austria, addressed polyintervention (including lifestyle) trials in the ongoing ASPIS (Austrian Stroke Prevention in Ischemic Stroke) trial (ISRNCT 01109836), which aims at a reduction of poststroke cognitive decline rates in mild stroke patients who are cognitively unimpaired at discharge. Patients are randomized to either normal treatment and follow-up or intense follow-up and guidance in establishing and maintaining a healthy lifestyle.

\section{Edo Richard, Amsterdam, The Netherlands, Spoke on Prevention of Dementia by Intensive Vascular Care}

Edo Richard, Amsterdam, the Netherlands, speaking on prevention of dementia by intensive vascular care, reported the ongoing cluster randomized controlled preDIVA trial that is performed in nondemented community-dwelling elderly $(n=3,534)$ individuals aged $70-78$ years. The intervention consists of multicomponent pharmacological and nonpharmacological treatment aimed at all vascular risk factors.

\section{Session VII: Reorganization and Networks after Stroke}

Reorganization of surviving brain networks is associated with functional improvement after stroke and this process is likely to be facilitated by neurorehabilitative training. Neuroimaging techniques are proving useful in the evaluation of novel treatments.

\section{Rick Dijkhuizen, Utrecht, The Netherlands, Described Longitudinal Alterations in Neuronal Networks Using Resting-State Functional MRI}

Rick Dijkhuizen, Utrecht, the Netherlands, described longitudinal alterations in neuronal networks after an experimental stroke model in rats using resting-state functional MRI and diffusion tensor imaging. Improvement of sensorimotor function was associated with wide-ranging changes in functional and structural connectivity within bilateral neuronal networks [17]. There was partial nor- malization of neuronal signal synchronization between the affected and unaffected sensorimotor cortices together with an increase in network efficiency. These modifications in cortical network organization were associated with an increase of initially declined fractional diffusion anisotropy in perilesional corticospinal tract regions. Correlation analyses revealed that improvement of sensorimotor performance scores were associated with restoration of ipsilesional corticospinal tracts, in combination with reinstatement of interhemispheric neuronal signal synchronization and normalization of cortical network organization.

\section{Nick Ward, London, United Kingdom, Discussed Brain Activity during Affected Hand Movement}

Nick Ward, London, United Kingdom, described how, in humans, increasing damage to the corticospinal system leads to a shift of functional MRI-measured brain activity during affected hand movement away from primary motor cortex (M1) toward premotor and supplementary motor areas [18]. In some patients, prominent activity also can be seen in the contralesional hemisphere. Analysis of connectivity shows that the influence of ipsilesional on contralesional M1 becomes more facilitatory in these patients. Furthermore, in some cases, the peak of corticomuscular coherence (measured using magnetoencephalography to indicate the cortical region directly contributing to motor execution) is seen in contralesional M1. These data support the idea that the brain is able to generate descending motor commands from a range of cortical motor areas in either hemisphere. Design of therapeutic cortical stimulation studies to enhance the effects of training need to take this into consideration.

\section{Andreas Luft, Zurich, Switzerland, Discussed the Role of Dopamine and Reward in Neurorehabilitation of Stroke}

After stroke, the brain is impaired in processing rewards potentially leading to deficits in reward-based learning. Destroying dopaminergic projections that most likely relay reward signals from the ventral tegmental area of the brain stem to primary motor cortex (M1) destroys learning in a rat model [19]. Subsequent substitution of dopamine into M1 restores learning. Interfering with intracortical dopaminergic signaling inside M1 destroys the ability of M1 neurons to undergo long-term potentiation, a basic cortical mechanism of motor skill learning. These 
data indicate that reward processing is integral to motor learning and, therefore, motor recovery. Enhancing the ability of stroke survivors to assess and process rewards has the potential to enhance motor recovery.

\section{Agnes Floel, Berlin, Germany, Described How 3 Days of Intensive Anomia Training in Chronic Aphasia Patients Led to Improvements}

Agnes Floel, Berlin, Germany, described how 3 days of intensive anomia training in chronic aphasia patients led to improvements in naming ability lasting at least 2 weeks after training [20]. Application of anodal transcranial direct current stimulation over the right temporo-parietal cortex enhanced the effect of training compared with cathodal transcranial direct current stimulation and sham stimulations. Subsequently, she applied anodal transcranial direct current stimulation over left ventral inferior frontal gyrus (a core language area) during functional MRI scanning of semantic word generation. Improved word retrieval during anodal transcranial direct current stimulation was paralleled by selectively reduced task-related activation in the left ventral inferior frontal gyrus and increased connectivity in major hubs of anatomically connected language areas. These results suggest that targeting specific brain regions within spared networks in combination with symptom-oriented treatment may help to promote recovery.

\section{Alex Leff, London, United Kingdom, Investigated the Effects of Behavioral Therapy Using Functional MRI in Chronic Aphasic Stroke Patients}

The speech perception of the patients improved with $50-100 \mathrm{~h}$ of phonological therapy. In the more severely affected group, the effects of therapy were correlated with changes in brain connectivity between layers of the auditory hierarchy [21]. A feedback connection (from right auditory cortex to right thalamus) was significantly strengthened, whereas a feedforward connection (from right auditory cortex to right planum temporale) was weakened. In other words, the feedback connections that code predictions have been improved by therapy, leading to smaller errors being passed on via the feedforward connections. These results illustrate the principle that connectivity parameters may be useful biomarkers for studying therapy effect.
According to an anonymous survey, the participants unanimously appreciated the endeavor of this first European workshop; $90-98 \%$ liked the selection of topics and speakers, the mixture of basic translational and clinical research, the scientific and social interactions, and the discussions. Many pointed out the academic noncommercial nature of the event and were enthusiastic to repeat the workshop on a regular basis.

\section{Disclosures}

H.C.D. has received honoraria for participation in clinical trials, contributions to advisory boards, or oral presentations from Abbott, Allergan, AstraZeneca, Bayer, Vital, BMS, Boehringer Ingelheim, CoAxia, Covidien, Daiichi-Sankyo, D-Pharm, EV3, Fresenius, GlaxoSmithKline, Janssen Cilag, Knoll, MSD, Medtronic, MindFrame, Neurobiological Technologies, Novartis, NovoNordisk, Paion, Parke-Davis, Pfizer, Sanofi-Aventis, ScheringPlough, Servier, Solvay, Thrombogenics, Wyeth, and Yamanouchi. Financial support for research projects was provided by AstraZeneca, GlaxoSmithKline, Boehringer Ingelheim, Lundbeck, Novartis, Janssen-Cilag, Sanofi-Aventis, Syngis, and Talecris. The Department of Neurology at the Duisburg-Essen University received research grants from the German Research Council (D.F.G.), German Ministry of Education and Research (B.M.B.F.), European Union, National Institutes of Health, Bertelsmann Foundation, and Heinz-Nixdorf Foundation. Within the past years, H.C.D. served as editor of Aktuelle Neurologie, Arzneimitteltherapie, Kopfschmerznews, Stroke News, and the Treatment Guidelines of the German Neurological Society, as coeditor of Cephalalgia, and on the editorial board of Lancet Neurology, European Neurology, and Cerebrovascular Diseases. D.L. has received honoraria for participation in clinical trials, contribution to advisory boards, or oral presentations from Allergan, Bayer, BMS, Boehringer Ingelheim, Braingate, Colucia Pharma, Daiichi Sankyo, Ebarre, Photothera, Sanofi-Aventis, Servier. N.W. has received research grants from the Wellcome Trust (grant 088414). 


\section{References}

1 Chamorro A, Urra X, Planas AM: Infection after acute ischemic stroke: a manifestation of brain-induced immunodepression. Stroke. 2007;38:1097-1103.

-2 Planas AM, Gómez-Choco M, Urra X, Gorina $\mathrm{R}$, Caballero M, Chamorro A: Brain-derived antigens in lymphoid tissue of patients with acute stroke. J Immunol. 2012;188: 2156-2163.

>3 Liesz A, Zhou W, Mracskó É, Karcher S, Bauer H, Schwarting S, et al: Inhibition of lymphocyte trafficking shields the brain against deleterious neuroinflammation after stroke. Brain. 2011;134:704-720.

4 Meisel C, Schwab JM, Prass K, Meisel A, Dirnagl U: Central nervous system injury-induced immune deficiency syndrome. Nat Rev Neurosci. 2005;6:775-786.

$>5$ Sarrafzadeh A, Schlenk F, Meisel A, Dreier J, Vajkoczy P, Meisel C: Immunodepression after aneurysmal subarachnoid hemorrhage. Stroke. 2011;42:53-58.

-6 Urra X, Cervera A, Obach V, Climent N, Planas AM, Chamorro A: Monocytes are major players in the prognosis and risk of infection after acute stroke. Stroke. 2009;40:12621268.

7 van de Beek D, Wijdicks EF, Vermeij FH, de Haan RJ, Prins JM, Spanjaard L, et al: Preventive antibiotics for infections in acute stroke: a systematic review and meta-analysis. Arch Neurol. 2009;66:1076-1081.
-8 Dirnagl U, Fisher M: International, multicenter randomized preclinical trials in translational stroke research: it's time to act. J Cereb Blood Flow Metab. 2012;32:933-935.

$\checkmark 9$ Foerch C, Montaner J, Furie KL, Ning MM, Lo EH: Invited article: searching for oracles? Blood biomarkers in acute stroke. Neurology. 2009;73:393-399.

10 Lansberg MG, Lee J, Christensen S, Straka M, De Silva DA, Mlynash M, et al: RAPID automated patient selection for reperfusion therapy: a pooled analysis of the Echoplanar Imaging Thrombolytic Evaluation Trial (EPITHET) and the Diffusion and Perfusion Imaging Evaluation for Understanding Stroke Evolution (DEFUSE) Study. Stroke. 2011;42:1608-1614.

11 International Stroke Genetics Consortium (ISGC), Wellcome Trust Case Control Consortium 2 (WTCCC2), Bellenguez B, Bevan S, Gschwendtner A, Spencer CA, et al. Genome-wide association study identifies a variant in HDAC9 associated with large vessel ischemic stroke. Nature Genetics. 2012; 44:328-333.

12 Foerch C, Niessner M, Back T, Bauerle M, De Marchis GM, Ferbert A, Grehl H, et al: Diagnostic accuracy of plasma glial fibrillary acidic protein for differentiating intracerebral hemorrhage and cerebral ischemia in patients with symptoms of acute stroke. Clin Chem. 2012;58:237-245.

-13 Brekenfeld C, Schroth G, Mordasini P, Fischer U, Mono ML, Weck A, et al: Impact of retrievable stents on acute ischemic stroke treatment. AJNR Am J Neuroradiol. 2011;32: 1269-1273.
14 Zhang Y, Tuomilehto J, Jousilahti P, Wang Y, Antikainen R, Hu G: Lifestyle factors on the risks of ischemic and hemorrhagic stroke. Arch Intern Med. 2011;171:1811-1818.

15 Strazzullo P, D’Elia L, Kandala NB, Cappuccio FP: Salt intake, stroke, and cardiovascular disease. BMJ. 2009;339:b4567.

16 Goldstein LB, Bushnell CD, Adams RJ, Appel LJ, Braun LT, Chaturvedi S, et al: Guidelines for the Primary Prevention of Stroke. A guideline for healthcare professionals from the American Heart Association/American Stroke Association. Stroke. 2011;42:517-584.

17 van Meer MP, Otte WM, van der Marel K, Nijboer CH, Kavelaars A, van der Sprenkel JW, et al: Extent of bilateral neuronal network reorganization and functional recovery in relation to stroke severity. J Neurosci. 2012;32:4495-4507.

18 Ward N: Assessment of cortical reorganisation for hand function after stroke. J Physiol. 2011;589:5625-5632.

19 Hosp JA, Pekanovic A, Rioult-Pedotti MS, Luft AR: Dopaminergic projections from midbrain to primary motor cortex mediate motor skill learning. J Neurosci. 2011;31: 2481-2487.

20 Floel A, Meinzer M, Kirstein R, Nijhof S, Deppe M, Knecht S, et al: Short-term anomia training and electrical brain stimulation. Stroke. 2011;42:2065-2067.

21 21. Schofield TM, Penny WD, Stephan KE, Crinion JT, Thompson AJ, Price CJ, et al: Changes in auditory feedback connections determine the severity of speech processing deficits after stroke. J Neurosci. 2012;32: 4260-4270. 